\title{
Production and investigation of heavy neutron rich nuclei
}

\author{
Sergey Zemlyanoy ${ }^{1, \star}, K_{\text {Konstantin Avvakumov }}{ }^{1}$, Eduard Kozulin $^{1}$, Valentin Fedosseev ${ }^{2}$, Robert Bark ${ }^{3}$, and Zenon \\ Janas $^{4}$ \\ 1 Joint Institute for Nuclear Research, 141980 Dubna, Russia \\ ${ }^{2}$ CERN, 1211, Geneva 23, Switzerland \\ ${ }^{3}$ iThemba LABS, Nat. Research Foundation, Somerset West 7129, South Africa \\ ${ }^{4}$ Faculty of Physics, University of Warsaw, Hoza 69, PL-00681 Warsaw,
}

\begin{abstract}
A project devoted to the production and study of neutron rich heavy nuclei (GALS - project) is being realized at Flerov Laboratory for Nuclear Reactions (FLNR) - JINR. GALS is planned to exploit available beams from the U-400M cyclotron in low energy multi-nucleon transfer reactions to study exotic neutronrich nuclei located in the "north-east" region of nuclear map. Products from 4.5 to $9 \mathrm{MeV} /$ nucleon heavy-ion collisions, such as ${ }^{136} \mathrm{Xe}$ on ${ }^{208} \mathrm{~Pb}$, are to be captured in a gas cell and selectively laser-ionized in a sextupole (quadrupole) ion guide extraction system.
\end{abstract}

\section{Introduction}

The closed neutron shell $\mathrm{N}=126$ - the so called last "waiting point" for the r-process - is located in the region of heavy neutron rich nuclei, "north-east" part of the nuclide chart, which is very important for nuclear physics investigations, especially for the understanding of astrophysical nucleosynthesis and r-process. (Fig. 1,2). Neutron shell $\mathrm{N}=126$ is a classical shell closures for nuclei along the line of stability. However, as one moves away from the line of stability, abrupt changes in nuclear structure can arise. Especially, appearance of new magic numbers and/or disappearance of traditional ones as well as the onset of deformation are expected, as e.g. already observed in the region of lighter nuclei.

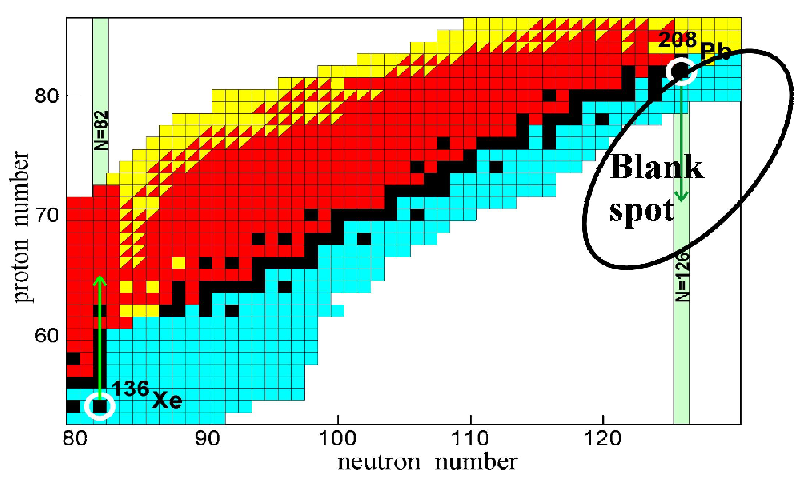

Figure 1. Upper "north-east" part of nuclear map.

Study of the structural properties of nuclei along the neutron shell $\mathrm{N}=126$ could contribute to the current discussion about the quenching of shell gaps in nuclei with

\footnotetext{
${ }^{\star}$ Corresponding author: e-mail: zemlya@jinr.ru
}

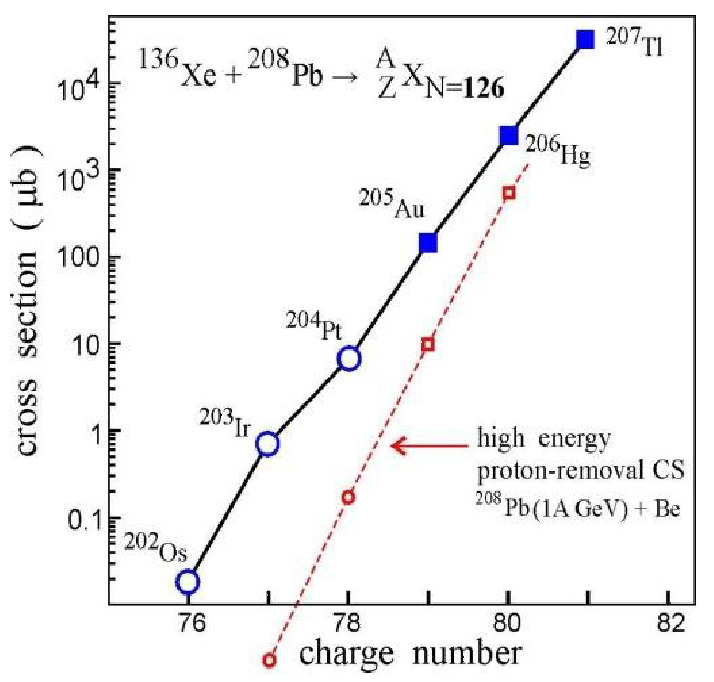

Figure 2. Cross sections for the formation of heavy nuclei in collisions of ${ }^{136} \mathrm{Xe}$ on ${ }^{208} \mathrm{~Pb}$ at center-of-mass energy of $700 \mathrm{MeV}$. Open circles indicate unknown isotopes [1]. The dashed curve illustrates the yield of the same isotopes in the proton removal reaction from closed shell nucleus ${ }^{208} \mathrm{~Pb}$.

large neutron excess. The creation and launch of this facility will open a new field of research in low-energy heavyion physics, and new horizons in the study of unexplored "north-east" area of the nuclear map. It could be helpful also for finding a new way for the production of heavy and superheavy nuclei.

Experiments at GALS are intended for production of heavy neutron rich nuclei in the above mentioned region by using multi-nucleon transfer reactions. As suggested by Zagrebaev and Greiner [1], several tens of new nuclides in 


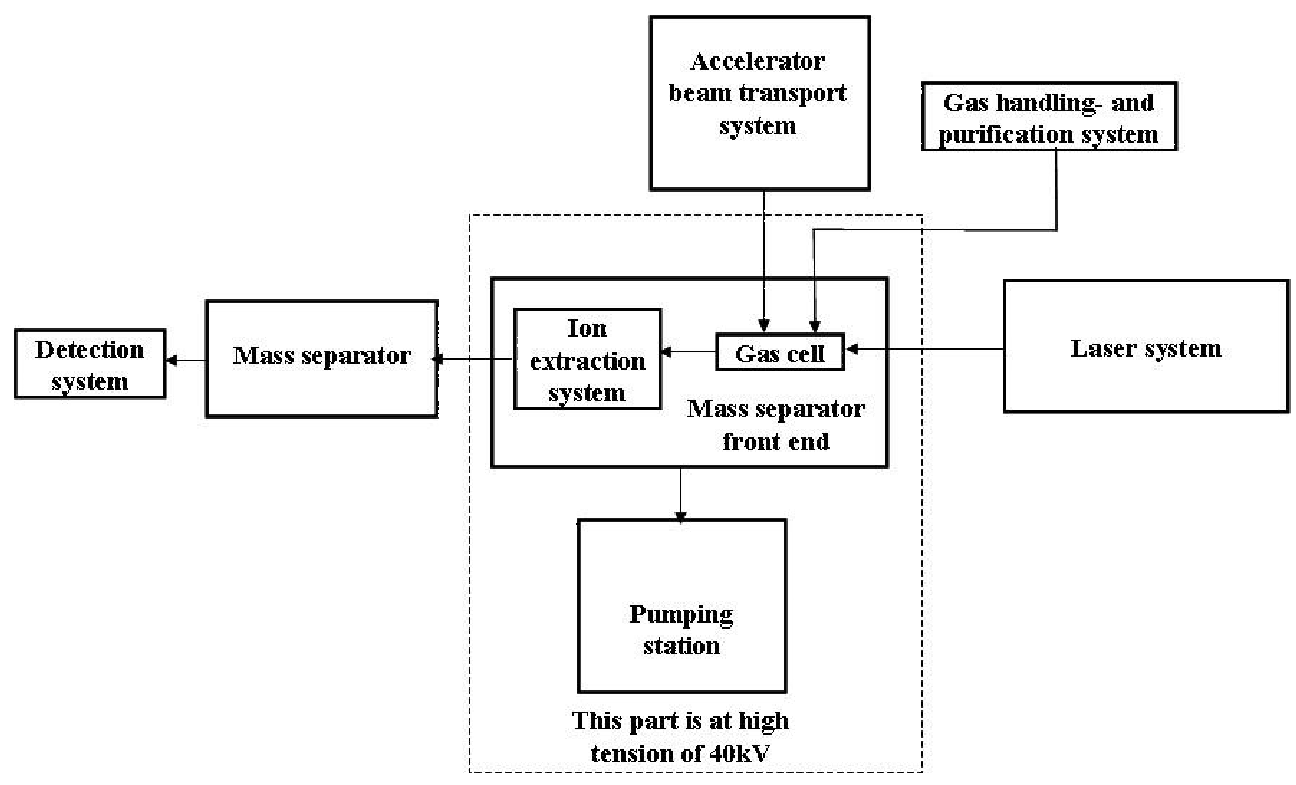

Figure 3. Block scheme of GALS-facilities.

the region of $\mathrm{N}=126$ and $Z \sim 75$ can be produced, for example, in the near-barrier collision of ${ }^{136} \mathrm{Xe}$ with ${ }^{208} \mathrm{~Pb}$. Even higher cross sections have been predicted for the production of new neutron rich nuclei in collisions of ${ }^{198} \mathrm{Pt}$ beam with ${ }^{238} \mathrm{U}$ target. At present this seems to be the only realistic method [1-3] that could fill the "blank spot" of the nuclear map (Fig. 1).

\section{Experimental method}

The experimental approach, fulfilling the purpose of this project, is a combined method of $Z$ and $\mathrm{A} / \mathrm{Q}$ separation. This method has been proposed in 1992 [4] and subsequently intensively studied (first at LISOL) and developed [5-13]. Known as In-Gas Laser Ionization and Spectroscopy (IGLIS), it is based on stopping of nuclear reaction products in a gas cell and subsequent selective resonance laser ionization [5-13]. This technique allows extracting nuclei with a given atomic number $Z$, while a separation of the single-ionized isotopes by their mass number can be done rather easily by a magnetic field.

Half-lives of heavy neutron rich nuclei of interest (as a rule, $\beta$-decaying), are much longer than the extraction time of ions from a gas cell. Detailed layout of the proposed facility can be found in $[2,3]$. Here we present only a brief description of the GALS facility (Fig. 3). Neutron rich isotopes of heavy elements are produced in multinucleon transfer reactions with heavy ions accelerated up to $5-10 \mathrm{MeV} /$ nucleon (depending on projectile-target combination). The target is a foil of about $300 \mu \mathrm{g} / \mathrm{cm}^{2}$ thickness (or larger). It is placed at the window of the gas cell (or inside it).

Nuclear reaction products recoiling out from the target as multi-charged ions are thermalized and neutralized by collisions with highly-purified argon or helium buffer gas. Then the atoms of interest (with a given $Z$ ) are ionized by means of two or three-step resonance laser irra- diation and are extracted by the gas flow through the exit into the vacuum chamber as singly charged ions $(\mathrm{Q}=+1)$ with low energies of about $0.2 \mathrm{eV}$. Subsequently, the ions are confined in a radiofrequency ion guide system, which allows pumping out the residual buffer gas while transporting the ions towards the mass separator. Then the ions are accelerated up to $30-60 \mathrm{keV}$ and selected by the massseparator. In this way a low-energy beam of singly-ionized ions with a good optical quality (small emittance, energy spread less than $1 \mathrm{eV}$ ) is produced. This allows obtaining typical mass resolution of 1500 after the dipole magnet. After mass separation a beam of radioactive ions with a definite atomic number and a previously chosen mass value is obtained. The background due to unwanted isobar and isotope admixture is significantly suppressed that leads to an enhanced sensitivity. This gives the possibility to perform subsequently high sensitive analysis of spectroscopic and decay properties of these nuclei, as well as measurements of their spins, magnetic dipole and electric quadrupole moments and charge radii by means of laser spectroscopy. The operation principle of the different parts of the set up can be found in a number of papers (see e.g. [14] and the references therein).

\section{Current status}

The ion beams available at FLNR fully satisfy the requirements for this project: the ions that can be used are quite different depending on the problem to be solved from ${ }^{16,18} \mathrm{O}$ to ${ }^{238} \mathrm{U}$, beam energies 4.5 - $9 \mathrm{MeV} /$ nucleon (slightly above the Coulomb barrier) and beam intensity up to $10^{13}$ pps are accessible with a beam size at the target of 3-10 $\mathrm{mm}$ and beam emittance of $20 \cdot \pi \cdot \mathrm{mm} \cdot \mathrm{mrad}$. Different heavy targets, including those of actinides, are expected to be used. Therefore the new facility can be developed and coupled directly to the available U-400M accelerator. The mass separator could be a standard magnet 
separator similar to GPS at ISOLDE II (see [2, 15]) but nevertheless substantial efforts are needed for its realization.

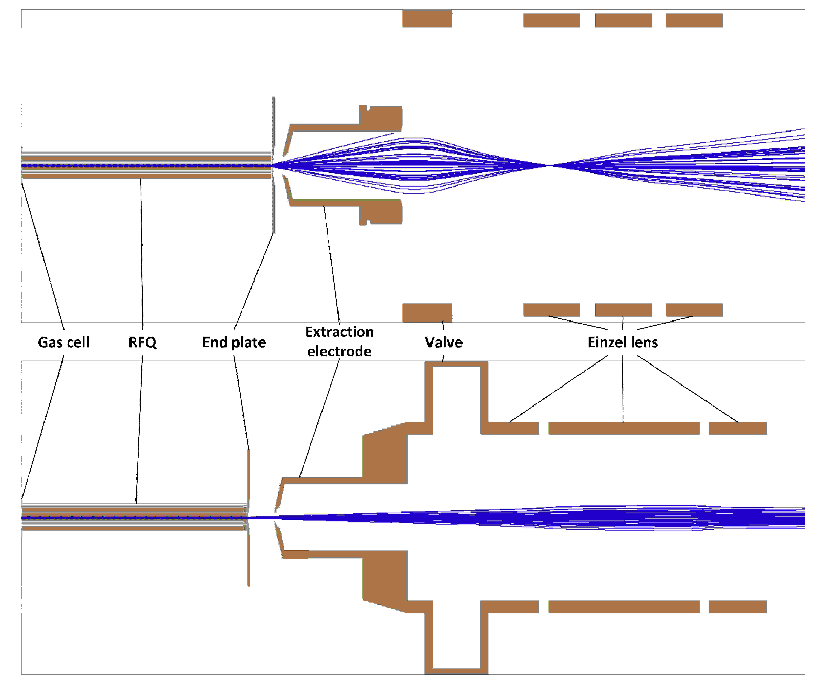

Figure 4. The LISOL-like setup (above) and the FLNR setup (below) in enlarged transverse scale with trajectories of the ions passing through.

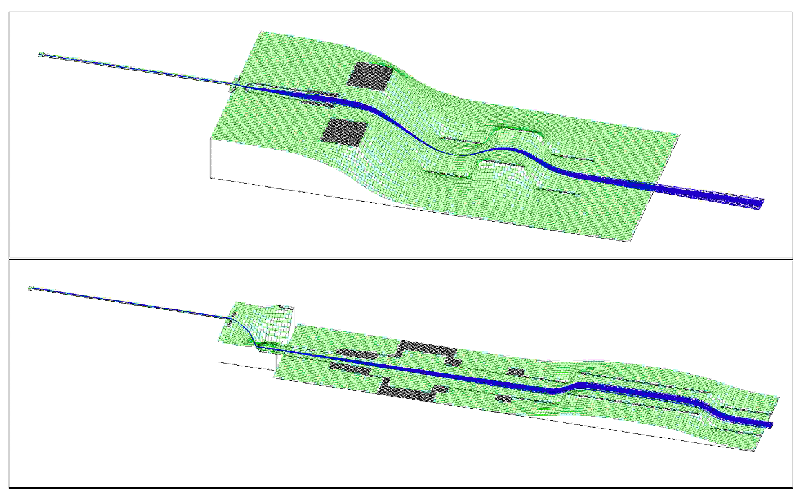

Figure 5. 4 The distribution of potential energy: above - LISOLlike; below - JINR.

\subsection{The ion extraction system simulation}

The ion extraction system is used for carrying the ions of interest (which are ${ }^{202} \mathrm{Os},{ }^{203} \mathrm{Ir},{ }^{204} \mathrm{Pt}$ with an electric charge of +1 ) coming out from the gas cell nozzle, accelerating them in an electric field and focusing in the detector system plane. Two system options were proposed:

1. The system of the existing LISOL setup in Leuven, Belgium.

2. The system designed in Flerov Laboratory of Nuclear Reactions, JINR, Dubna.

The systems are very similar and contain the same main elements, Fig. 3. The differences are the geometries after the extraction electrode and the voltages applied to the extraction electrode and the end plate $\left(U_{E E}=0\right.$ $\mathrm{V}, U_{E P}=39.54 \mathrm{kV}$ in the JINR system, and $U_{E E}=38.34$
Table 1. Output ions kinetic energy parameters and overall electrostatic system efficiencies (without considering gas influence) at the different types of ion guide system. Statistics of 1000 ions used in each case.

\begin{tabular}{|c|c|c|}
\hline LISOL-based system & $\mathrm{SPIG}, \mathrm{U}_{\mathrm{rf}}=150 \mathrm{~V}$ & $\begin{array}{l}\text { Quadrupole ion } \\
\text { guide, } \mathrm{U}_{\mathrm{rf}}=159 \mathrm{~V}\end{array}$ \\
\hline $\begin{array}{l}\text { Gas cell exit hole } \\
\text { diameter }\end{array}$ & $0.5 \mathrm{~mm}$ & $0.5 \mathrm{~mm}$ \\
\hline Mean value, eV & 39707.5539710 .78 & 39710.7839709 .99 \\
\hline Standard deviation, $\mathrm{eV}$ & 8.22 & 6.14 \\
\hline Efficiency, \% & 98 & 99 \\
\hline FLNR system & $\mathrm{SPIG}, \mathrm{U}_{\mathrm{rf}}=150 \mathrm{~V}$ & $\begin{array}{l}\text { Quadrupole ion } \\
\text { guide, } U_{\mathrm{rf}}=159 \mathrm{~V}\end{array}$ \\
\hline $\begin{array}{l}\text { Gas cell exit hole } \\
\text { diameter }\end{array}$ & $0.5 \mathrm{~mm}$ & $0.5 \mathrm{~mm}$ \\
\hline Mean value, eV & 38481.8838484 .78 & 38484.7838485 .67 \\
\hline Standard deviation, $\mathrm{eV}$ & 5.06 & 5.67 \\
\hline Efficiency, \% & 100 & 100 \\
\hline
\end{tabular}

$\mathrm{kV}, U_{E P}=39.75 \mathrm{kV}$ in a LISOL-like system). An SPIG (sextupole ion guide), or quadrupole ion guide as an option, is the same in both systems and has on its rods a voltage up to $500 \mathrm{~V}$ and a frequency of $4.7 \mathrm{MHz}$. Both of the systems were simulated using SIMION 8.0 package (http://simion.com/). The initial speed of the singly charged ions of ${ }^{204} \mathrm{Pt}$ at the nozzle exit was set to $560 \mathrm{~m} / \mathrm{s}$, which corresponds to kinetic energy of $0.332 \mathrm{eV}$. The simulation results are shown in Fig. 4, 5 and Table 1.

In the LISOL-based setup beam divergence after the end plate is significantly higher than in FLNR setup. This leads to beam losses in collisions with the inner surface of the extraction electrode (about 1\% of particles were lost there in LISOL-based setup model compared to no losses in the FLNR setup model).

Moreover, the voltage of $20 \mathrm{kV}$ on the middle Einzel lens electrode in the LISOL-based setup is not enough to focus the whole output beam (the voltage of about $35 \mathrm{kV}$ could be needed). The main acceleration gap in the FLNR setup is between the end plate and the extraction electrode which results to better collimated beam through the extraction electrode and the Einzel lens. On the other hand, such a high electric field $(10 \mathrm{kV} / \mathrm{cm})$ could increase the possibility of an electric breakdown. The gas pressure in that region of the vacuum chamber is about $10^{-6}$ Torr, but in case of gas cell foil breakthrough it can increase significantly. It was calculated that in case of adiabatic argon expansion the pressure in this region would be still less than $1.5 \cdot 10^{-3}$ Torr which, according to the Paschen curves (the distance between the electrodes is $4 \mathrm{~cm}$ ), remains in safe from electric breakdown area. Yet, some additional safety measures should be taken.

The efficiencies (see Table 1) were calculated without taking into account residual gas influence. Basing on the LISOL SPIG efficiency of $66 \%$ [8], we can expect the efficiency of the FLNR system with SPIG to be $67 \%$ or higher. Thus, the system developed in FLNR looks more preferable due to the less beam losses and less ion energy dispersion. 
Table 2. Laser to be used in multistep ionization. The lasers already delivered and installed are presented in bold.

\begin{tabular}{lllll}
\hline Type & $\begin{array}{l}\text { Output power } \\
\text { main\&harmonic, } \\
\text { W } \\
(2 \mathrm{nd}), 3 \mathrm{rd}, \text { 4th }\end{array}$ & $\begin{array}{l}\text { Puls } \\
\text { frequency, } \\
\mathrm{Hz}\end{array}$ & $\begin{array}{l}\text { Puls } \\
\text { length, } \\
\mathrm{ns}\end{array}$ & $\begin{array}{l}\text { Wave } \\
\text { length, } \\
\mathrm{nm}\end{array}$ \\
\hline Dye laser & $\mathbf{3 , ( 0 . 3 )}$ & $\mathbf{1 0}^{4}$ & $\mathbf{1 0 - 3 0}$ & $\mathbf{2 1 3 - 8 5 0}$ \\
Ti:Sapphire & $2,(0.2), 0.04$ & $10^{4}$ & $30-50$ & $680-960$ \\
Nd-YAG & $\mathbf{( 8 0 - 1 0 0 ) , 2 0 - 4 0}$ & $\mathbf{1 0}^{4}$ & $\mathbf{1 0 - 5 0}$ & $\mathbf{5 3 2}$ \\
Matisse & & & & \\
system & & & & \\
Ring dye & $0.8-6$ & $\mathrm{cw}$ & $\mathrm{cw}$ & $540-900$ \\
Ti:Sapphire & $0.8-6.5$ & $\mathrm{cw}$ & $\mathrm{cw}$ & $700-1000$ \\
\hline
\end{tabular}

\subsection{Laser system and excitation schemes}

The choice of specific laser ionization scheme, the type and number of lasers is determined by the ionization potentials and and the atomic level schemes of the elements under study. In our case a three-step scheme of ionization looks more favorable. Such a scheme allows choosing more effective optical transitions to increase the yield of resonance-ionized ions, although the use of two-step ionization scheme is as not excluded. Dye laser systems pumped by second and third harmonics of Nd:YAG can provide tuning in a broad spectral range: from near UV to near IR [16]. The generation of a Ti:Sapphire lasers, shifted to the red and infrared edge of the spectrum (680$960 \mathrm{~nm}$ ), can be also used as complementary to that of the dye lasers. Thus, the installation of two laser-ionization schemes-with dye lasers and Ti:Sapphire lasers - would be of a great benefit allowing to meet diverse experimental demands. Such a double RILIS scheme is already realized in CERN [17]. The parameters of the delivered (and already installed) laser as well as of the ordered ones are summarized in Table 2.

At the first stage of our experiments, excitation schemes using 3-step ionization with a non-resonant transition to continuum will be tested with the available lasers: two dye lasers Credo (Sirah) and Nd:YAG laser. The Credo laser has maximal average power of $20 \mathrm{~W}$ at fundamental wavelength and $2 \mathrm{~W}$ at the second harmonics; its line width is $1.8 \mathrm{GHz}$ at pulse duration about $7 \mathrm{~ns}$. It is an option that allows remote control of wavelength with stabilization to an external laser wavelength meter. The $\mathrm{Nd}$ :YAG laser (product of Edge Wave $\mathrm{GmbH}$ ) generates maximal average power of $90 \mathrm{~W}$ and $36 \mathrm{~W}$ for the second and third harmonic respectively with repetition rate $10-15$ $\mathrm{kHz}$ and pulse duration of $8-10 \mathrm{~ns}$. The divergence parameter of the green beam is $\mathrm{m}^{2}=1.4$. It needs electrical power supply of $3.6 \mathrm{~kW}$ including $1.6 \mathrm{~kW}$ for the water chiller.

A Matisse series of a ring cw-dye laser and cwTi:Sapphire laser pumped by cw-Nd:YAG laser is planned as a seeding system. Such a system has an advantage in the cases when tuning of the laser wavelength is necessary, e.g. for laser spectroscopy.

As osmium is one of most prominent candidates to achieve the neutron magic line $\mathrm{N}=126$ we have checked ionization schemes of this element (Table 3). Although there are many observed spectral lines, as the atomic spec- tra information is not yet complete: most of the transitions are not assigned [16-19] and the atomic strengths are not known. Nevertheless several of the possible RIS scheme for Os have been previously studied [20,21]. The most favorable ionization scheme is expected to be the resonance three steps one via an auto-ionizing state. At present, no literature information is available about such states except the one at $71032 \mathrm{~cm}^{-1}$ corresponding to the third step of case C [20]. For this reason at the second stage of the GALS activities a search for auto-ionizing states is planned. At the next phase of experiments, an off-line study of the optimal ionization scheme will be conducted. It supposes a detailed experimental study the efficiency of the different ionization schemes.

\section{Outlook}

A new GALS facility at FLNR cyclotron U-400M is in a stage of build-up. GALS will apply the highly selective and efficient technique of stepwise resonant ionization in a gas cell (of the element of interest) with subsequently mass separation. In fact, it is a mass separator from a new type as yet not used in JINR. GALS is addressed to the production and study of new neutron rich nuclei located along the neutron shell $\mathrm{N}=126$. This is the so called "north-east" part of the nuclear map. Nuclei from this region are as yet "terra incognita" but of high fundamental interest mainly due to their large impact of on the astrophysical r-process. The most efficient method to produce such nuclei, as motivated in [1], are the multi-nucleon transfer reactions. Our estimations show that at target thickness $0.3 \mathrm{mg} / \mathrm{cm}$, ion beam of $0.1 \mathrm{p} \mu \mathrm{A}$ and setup efficiency of $10 \%$ we would be able to measure decay properties of 1 new isotope per day.

\section{References}

[1] V. Zagrebaev and W. Greiner, Phys. Rev. Lett. 101, 122701 (2008)

[2] V. I. Zagrebaev, S. G. Zemlyanoy, E. M. Kozulin et al., Hyperfine Interacs. 227, 181 (2014)

[3] V. I. Zagrebaev, S. G. Zemlyanoy, E. M. Kozulin et al., J. Phys.Conf. Series 533, 012025 (2014)

[4] P. Van Duppen, P. Dendooven, M. Huyse et al., Hypfine Interacs. 74, 193 (1992)

[5] L. Vermeeren, N. Bijnens, M. Huyse et al., Phys. Rev. Lett. 73, 1935 (1994)

[6] Y. Kudryavtsev et al., Nucl. Instrum. Methods Phys. Res. B 114, 350 (1996)

[7] Y. Kudryavtsev et al., Nucl. Instrum. Methods Phys. Res. B 204, 336 (2003)

[8] M. Facina et al., Nucl. Instrum. Methods B 226, 401 (2004)

[9] I. D. Moore et al., J. Phys. G: Nucl. Part. Phys. 31, S1499 (2005)

[10] Y. Kudryavtsev et al., Nucl. Instrum. Methods Phys. Res. B 267, 2908 (2009)

[11] R. Ferrer et al., Nucl. Instrum. Methods Phys. Res. B 291, 29 (2012) 
Table 3. Suggested two $\left(\lambda_{3}=\lambda_{1}-\right.$ case A or $\lambda_{3}=\lambda_{2}$ - case B) and three step (case C) ionization schemes of OsI.

\begin{tabular}{|c|c|c|c|c|c|c|c|c|}
\hline$\lambda_{1}$ (air), $\AA$ & $E_{1}, \mathrm{~cm}^{-1}$ & State I & $J_{1}$ & $\lambda_{2}$ (air), $\AA$ & $E_{2}, \mathrm{~cm}^{-1}$ & State II & $J_{1}$ & case \\
\hline 2909.06 & 34365.33 & $6 s 6 p^{5} F^{0}$ & 5 & 4752.16 & 55402.47 & $6 s 7 s ?$ & $?$ & $\mathrm{C}$ \\
\hline 3018.04 & 33124.48 & $6 s 6 p^{7} P^{0}$ & 3 & 5580.66 & 51038.49 & $6 s 7 s e^{5} D$ & 0 & $A$ or $B$ \\
\hline 3267.94 & 30591.45 & $6 s 6 p^{7} P^{0}$ & 4 & 5509.33 & 48737.34 & unknown & 4 & A \\
\hline 3301.56 & 30279.95 & $6 s 6 p^{7} F^{0}$ & 5 & 4815.96 & 51038.49 & $6 s 7 s e^{5} \mathrm{D}$ & 4 & A or $B$ \\
\hline 4260.85 & 23462.90 & $6 s 6 p^{7} D^{0}$ & 5 & 3157.24 & 55127.00 & unknown & $?$ & $A$ or $B$ \\
\hline 4420.47 & 22615.69 & $6 s 6 p^{7} D^{0}$ & 4 & 4066.69 & 48737.44 & $6 s 7 s e^{7} \mathrm{D}$ & 4 & A or B \\
\hline 4420.47 & 22615.69 & $6 s 6 p^{7} D^{0}$ & 4 & 3827.14 & 47198.7 & $6 s 7 \mathrm{~s} \mathrm{e}^{7} \mathrm{D}$ & 5 & A or $B$ \\
\hline
\end{tabular}

[12] R. Ferrer et al., Nucl. Instrum. Methods Phys. Res. B 317, 570 (2013)

[13] Y. Kudryavtsev et al., Nucl. Instrum. Methods Phys. Res. B 297, 7 (2013)

[14] P. Campbell, I. D. Moore, M. R. Pearson, Prog. Particle Nucl. Phys. 86, 127 (2016)

[15] H. L. Ravn and B. W. Allardyce In: Treatise on Heavy-Ion Science, 8, 363 (1989)

[16] V. N. Fedosseev, L. E. Berg, D. V. Fedorov et al., Rev. Sci. Instrum. 83, 02 A903 (2012)

[17] S. Rothe, B. A. Marsh, C. Mattolat et al., J. Phys.: Conf. Series 312, 052020 (2011)

[18] C. H. Corliss, W. R. Bozman, Experimentsl transition probabilities for spectral lines of seventy elements,
(NBS Monograph 53 1962)

[19] W. F. Meggers, C. H. Corliss, B. F. Scribner: Tables of spectral lines intensities (second edition), (NBS Monograph 145 1975)

[20] https://www.nist.gov/pml/atomic-spectra-database (version 5, updated October 2015)

[21] J. E. Sansonetti, W.C. Martin, Handbook of Basic Atomic Spectroscopic Data. J. Phys. Chem. Reference Data 34, 1559 (2005)

[22] J. D. Blum, M. J., Pellin, W. F. Calaway, et al., Anal. Chem. 62, 209 (1990)

[23] W. F. Calaway, R. C. Wien, D. S. Burnett, et al., J. Vac. Sci. Technol. A 13, 1310 (1995) 